\title{
High-Lift Propeller System Configuration Selection for NASA's SCEPTOR Distributed Electric Propulsion Flight Demonstrator
}

\author{
Michael D. Patterson, ${ }^{*}$ Joseph M. Derlaga ${ }^{\dagger}$ and Nicholas K. Borer, ${ }^{\ddagger}$ \\ NASA Langley Research Center, Hampton, VA, 23681
}

\begin{abstract}
Although the primary function of propellers is typically to produce thrust, aircraft equipped with distributed electric propulsion (DEP) may utilize propellers whose main purpose is to act as a form of high-lift device. These "high-lift propellers" can be placed upstream of wing such that, when the higher-velocity flow in the propellers' slipstreams interacts with the wing, the lift is increased. This technique is a main design feature of a new NASA advanced design project called Scalable Convergent Electric Propulsion Technology Operations Research (SCEPTOR). The goal of the SCEPTOR project is design, build, and fly a DEP aircraft to demonstrate that such an aircraft can be much more efficient than conventional designs. This paper provides details into the high-lift propeller system configuration selection for the SCEPTOR flight demonstrator. The methods used in the high-lift propeller system conceptual design and the tradeoffs considered in selecting the number of propellers are discussed.
\end{abstract}

\section{Nomenclature}

$c \quad$ chord length

$C_{i} \quad$ coefficient row vector for $\beta$ surrogate model

$c_{l} \quad$ section lift coefficient

$C_{D 0} \quad$ parasite drag coefficient

$c_{i, j} \quad$ scalar coefficient value for $\beta$ surrogate model

D drag

$i_{p} \quad$ propeller slipstream inclination angle

$K_{L} \quad$ lift multiplier (i.e., ratio of blown to unblown lift)

$L \quad$ lift

$L^{\prime} \quad$ lift per unit span

$R \quad$ radius

$T$ thrust

$u \quad$ distance of disk upstream of wing leading edge

$V \quad$ velocity

$w \quad$ induced velocity from point vortex

$X \quad$ column vector for $\beta$ surrogate model, $\left[\begin{array}{llllll}1 & u / c & (u / c)^{2} & (u / c)\left(V_{j} / V_{\infty}\right) & \left(V_{j} / V_{\infty}\right) & \left(V_{j} / V_{\infty}\right)^{2}\end{array}\right]^{T}$

$\alpha \quad$ angle of attack

$\beta \quad$ velocity multiplier or twist angle of local blade element

$\Gamma \quad$ circulation

$\rho \quad$ density

$\theta_{\text {swirl }} \quad$ swirl angle

\footnotetext{
*Aerospace Engineer, Aeronautics Systems Analysis Branch, 1 N. Dryden St. MS 442, AIAA Member.

${ }^{\dagger}$ Research Scientist, Computational AeroSciences Branch, 15 Langley Boulevard MS 128, AIAA Member.

${ }_{\ddagger}^{\ddagger}$ Technical Lead, Aeronautics Systems Analysis Branch, 1 N. Dryden St. MS 442, AIAA Senior Member.
} 


\begin{tabular}{ll}
\multicolumn{2}{l}{ Subscripts } \\
$\infty$ & freestream \\
$a$ & axial or absolute \\
$e p$ & effective value aft of the propeller \\
$j$ & jet \\
$p$ & propeller \\
$t$ & tangential
\end{tabular}

\section{Introduction}

Over the past several years, NASA has been studying the impacts of electric propulsion technologies on aircraft design. Electric motors have drastically different characteristics than conventional internal combustion or turbine engines including incredibly high efficiencies (above 90\%), very small volumes, low weights, few moving parts, no shaft power lapse with altitude, and the ability to scale over a wide range of sizes without losses in efficiency or power-to-weight ratio. ${ }^{1}$ These new characteristics open up new degrees of freedom for aircraft designers; specifically, designers can place these motors in many different locations to achieve a synergistic integration of the propulsion system with the rest of the airframe in a strategy generally called distributed electric propulsion (DEP). One potentially advantageous DEP integration strategy is illustrated in Fig. 1. This configuration employs two types of propellers: many "high-lift propellers" distributed

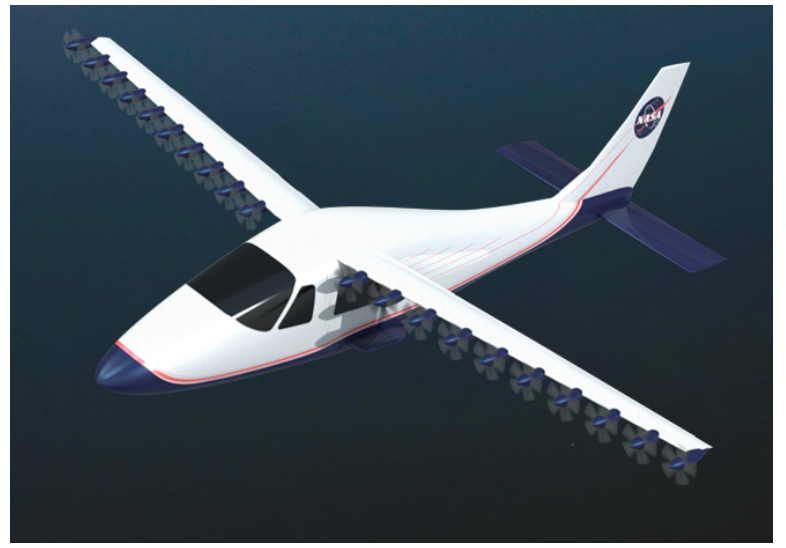

(a) Low-speed propeller configuration

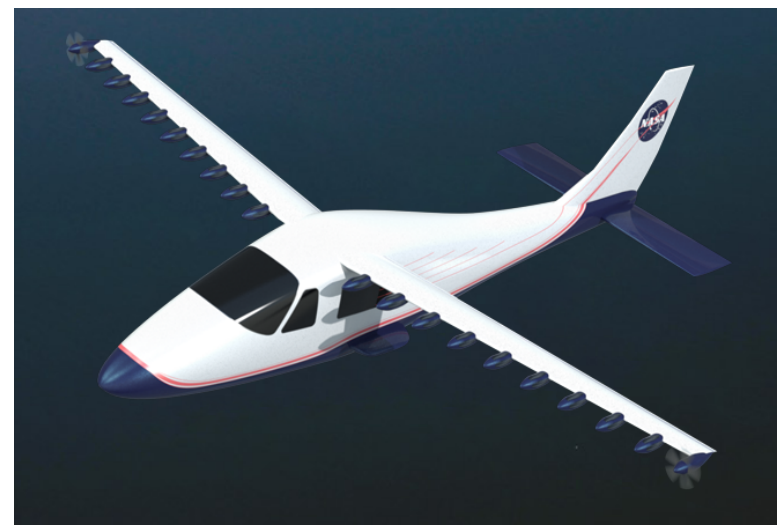

(b) High-speed propeller configuration

Figure 1. NASA Leading Edge Asynchronous Propellers Technology (LEAPTech) distributed electric propulsion concept proposed by Moore and Fredericks ${ }^{1}$

upstream of the wing's leading edge and two cruise propellers - one at each wing tip. The high-lift propellers act as a form of high-lift device by increasing the dynamic pressure over the sections of the wing in the props' slipstreams, which increases the total lift produced. Just like conventional high-lift devices, ${ }^{a}$ these props are only operated at low speeds as shown in Fig. 1(a); during higher-speed flight, the high-lift props are folded and stowed against the nacelles to reduce drag as shown in Fig. 1(b). The two cruise propellers provide all the propulsive thrust for the aircraft during higher-speed flight. By placing these props at the wing tips, they not only produce thrust but also act to reduce the induced drag of the configuration through beneficial interactions with the wing tip vortex. ${ }^{2,3}$

NASA is currently designing a flight demonstrator aircraft similar to that shown in Fig. 1 to demonstrate the potential efficiency gains that can be realized by such a configuration. This project, called Scalable Convergent Electric Propulsion Technology Operations Research (SCEPTOR), is retrofitting a Tecnam P2006T aircraft with a new, smaller wing that employs high-lift and tip propellers with the goal of creating a $350 \%$ to $500 \%$ more efficient aircraft in cruise. Although some may be skeptical of such large efficiency gains, it is important to realize that nearly $300 \%$ of the improvement can be credited solely to the move from an internal combustion engine of approximately $30 \%$ efficiency to electric motors with efficiencies of more than $90 \%$. The rest of the efficiency gains can be achieved from wisely distributing the electric motor-driven

\footnotetext{
${ }^{\mathrm{a}}$ e.g., flaps
} 
propellers across the wing. Specifically, the tip propellers reduce the aircraft's induced drag, and the wing size can be greatly reduced while still achieving the ability to stall at the same speed as the baseline Tecnam P2006T through the use of high-lift propellers. The original Tecnam P2006T aircraft has a wing loading of approximately $17 \mathrm{lb} / \mathrm{ft}^{2}$, which enables it to stall at approximately 55 knots; however, this high wing loading is inefficient for the aircraft's high-speed cruise condition of approximately 135 knots at 8,000 ft. The new wing being designed in the SCEPTOR project will have a $45 \mathrm{lb} / \mathrm{ft}^{2}$ wing loading to enable much more efficient cruise flight at a 150 knot speed at 8,000 ft.

The key to increasing the wing loading is to create a high-lift system that can provide the required lift at low speeds. The SCEPTOR aircraft will employ a high-lift system consisting of a single hinge point, slotted flap and high-lift propellers. This high-lift system is being designed to provide aircraft $C_{L_{\max }}$ values of approximately 4.4 at 55 knots. This paper will explain the process used to design the high-lift propellers. We will discuss how the number of high-lift propellers and initial design of these props was selected.

\section{A. A Brief Overview of Propeller-Wing Interaction}

This section provides an overview of the impacts of tractor propellers on downstream wings. An understanding of this general propeller-wing interaction is necessary for analyzing the impact of high-lift propellers on wings. A thorough review of the topic is provided by Veldhuis. ${ }^{4}$

The helical vortex system produced by propeller blades creates a complicated, non-uniform flowfield aft of the propeller. Generally speaking, the velocity varies along the propeller radius and around the circumference of the swept disk with time. The velocity at any point aft of the propeller can be in any arbitrary direction, but it is primarily directed along the axis of the propeller and tangent to that axis.

The axial velocity induced by the propeller (i.e., that directed along the propeller axis) is often the largest induced velocity component. Per momentum theory principles, this is the only velocity component that is actually desirable since the thrust is proportional to the axial velocity increase. The propeller begins to accelerate flow in the axial direction upstream of the disk, and this acceleration continues as the flow moves downstream of the disk.

The tangential component of velocity, which is often called swirl, is caused by a combination of the trailing vortex system and the viscous drag of the blades. Unlike the axial velocity, the propeller will induce no swirl upstream of the prop; rather, the tangential velocity is first generated at the propeller disk and remains approximately constant moving aft in the slipstream (if viscous dissipation effects are ignored). Since this velocity is not useful for producing thrust, it is generally desired to reduce the swirl imparted to the flow. For quantifying the amount of swirl in the slipstream,

The swirl angle, $\theta_{\text {swirl }}$, can be determined from Eq. 1 for any location in the slipstream.

$$
\theta_{\text {swirl }}=\tan ^{-1}\left(\frac{V_{t}}{V_{\infty}+V_{a}}\right)
$$

This angle is useful in appreciating the degree to which the local flow direction deviates from the desired purely axial direction. Though the angle varies with radial and axial locations, a single average value far downstream is presented in the results below.

The wing sections will react to the flow in the propeller slipstream much like they would to a freestream flow of equal velocity and effective angle of attack. The swirl induced by the propeller tends to increase the angle of attack of wing sections aft of the upward moving half of the propeller plane and decrease the angle of attack of those wing sections downstream of the downward moving side. This leads to large "peaks and valleys" in the wing lift distribution. The induced axial velocity from the propeller tends to increase the apparent velocity over the wing, which generally increases the lift. This is, in fact, the very purpose of high-lift propellers.

The general impacts of propeller slipstreams on wing lift distributions are shown in Fig. 2, which is reproduced from Ref. 4. As shown in the figure, the lift of the wing sections downstream of the upward moving side of the propeller disk is markedly increased due to both the swirl and axial velocity increases in the slipstream. The lift aft of the downward moving side of the propeller disk is reduced from the baseline distribution due to the swirl, but it is not decreased as much as the opposite side is increased due to the axial velocity increase in the flow there. 


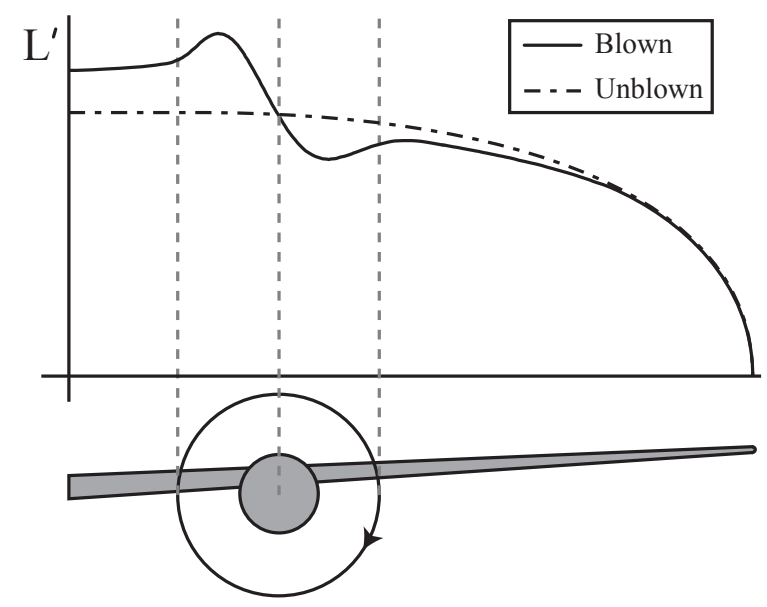

Figure 2. Generic lift distribution of a blown and unblown wing showing the general impacts of the propeller slipstream velocities [reproduced from Ref. 4]

\section{Analysis Methods}

Because the interactions of a propeller with a downstream wing are complex, there have been many different manners of modeling the impacts of propeller-wing interaction. Many methods are built on lifting line theory or lifting surface methods and require representation of the wing lift distribution via semi-infinite series of Fourier coefficients. ${ }^{5,6,7,8,9,10,11}$ For the purposes of the design space explorations of interest here, analysis methods that execute rapidly and provide insight to the designer of high-lift propellers are desired; the aggregate lift augmentation from the propeller(s) is of interest and detailed lift distributions are of lesser importance. Consequently, an extension of the method presented by Patterson and German ${ }^{12}$ will be employed for this paper. The general method is briefly described below and then a modification to the method is presented.

\section{A. General Lift Augmentation Model}

The simplified model for predicting the lift increase from propellers presented by Patterson and German is built on considerations of thin-wing theory and assumes incompressible flow. It is derived from a twodimensional model of the wing-propeller combination that has the geometry shown in Fig. 3. The airfoil is

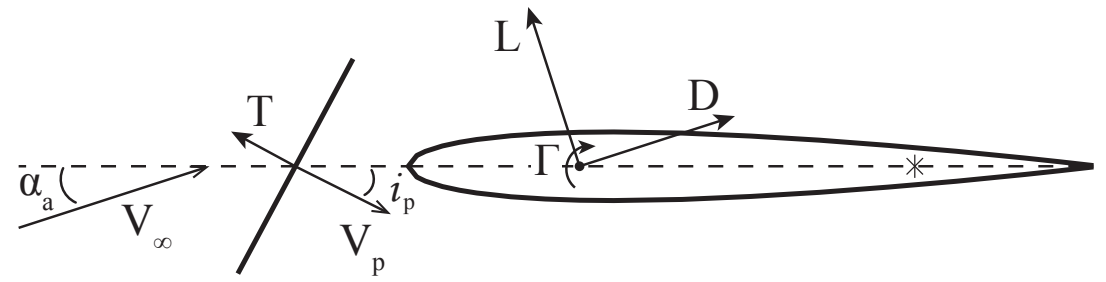

Figure 3. The geometry describing a two dimensional point vortex representation of an airfoil and the incoming local frestream velocity and propeller slipstream velocity relative to the local airfoil zero-lift line

considered to be completely immersed in the slipstream from the propeller so that the velocity experienced at the airfoil, $\boldsymbol{V}_{\boldsymbol{e p}}$, is equivalent to the vector sum of the freestream, $\boldsymbol{V}_{\infty}$ and the velocity induced by the propeller, $\boldsymbol{V}_{\boldsymbol{p}}$. The airfoil is represented by a single point vortex of circulation strength $\Gamma$, which is placed at the quarter chord point on the zero-lift line. ${ }^{\mathrm{b}}$ The point vortex induces a velocity, $w$, downward and normal

\footnotetext{
${ }^{\mathrm{b}}$ Note that Fig. 3 shows a symmetric airfoil where the zero-lift line and the chord line are coincident. For positively cambered airfoils, the absolute angle of attack, $\alpha_{a}$, is increased over the geometric angle of attack of the chord line.
} 
to the zero-lift line at a control point located at the $3 / 4$ chord point of the zero-lift line, which is denoted by an asterisk $(*)$ in Fig. 3. The circulation strength is determined by requiring flow tangency at the control point. The lift per unit span of the airfoil section is calculated via the Kutta-Joukowski theorem with the velocity, $V_{e p}$, and the circulation strength, $\Gamma$ (i.e., $L^{\prime}=\rho V_{e p} \Gamma$ ).

Ultimately, this model determines the percentage increase in the lift generated by the airfoil section (i.e., $\left.\Delta L^{\prime} / L_{\infty}^{\prime}\right)$ through consideration of the changes in circulation and local velocity induced by the propeller's slipstream relative to the circulation and lift without propeller blowing. The model predicts that the slipstream orientation relative to the airfoil (i.e., the magnitude of the angle $i_{p}$ ) can have large impacts on the circulation strength and lift.

A vector diagram depicting the flow aft of the propeller in an exemplary situation similar to that depicted in Fig. 3 is shown in Fig. 4. In order to maintain flow tangency at the control point, the circulation must

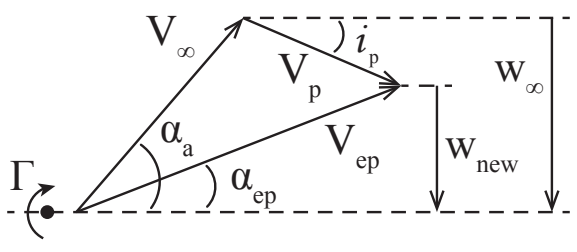

Figure 4. Vector diagrams showing the effective angle of attack and required induced velocity from a point vortex for various propeller installation angles (showing here a case where the propeller is inclined upward)

induce a downwash of $w_{\infty}$ when only the freestream velocity is present and a downwash of $w_{\text {new }}$ when propeller blowing is added.

However, simply considering the geometry depicted above will generally over-predict the lift increase. This can be explained by considering the geometry shown in Fig. 5 where a flat plate (or more generally, an airfoil) is placed in a "jet" of finite height. If the jet velocity is caused by a propeller, then $V_{j}=V_{e p}=V_{\infty}+V_{p}$ following the notation above. The point vortex model predicts that in the situation shown in Fig. 5, the lift

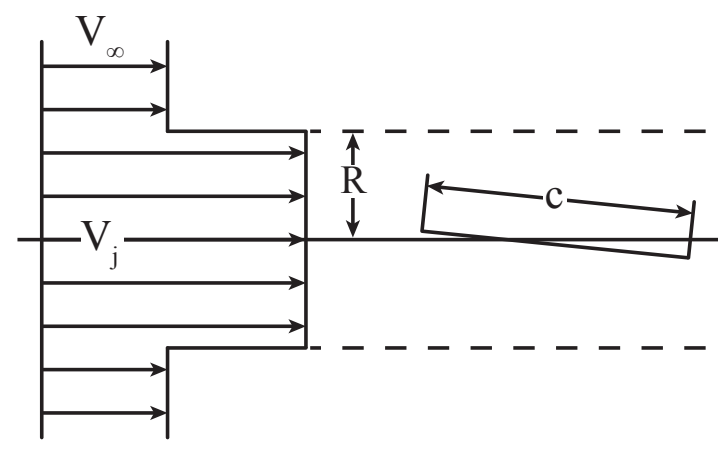

Figure 5. A flat plate airfoil placed in a slipstream of finite height

over the airfoil should be equivalent to the lift produced by the airfoil in a flow with freestream velocity $V_{j}$. However, other researchers have shown that for situations where $R$ (i.e., the height of the jet) is finite, the lift produced is less than if the airfoil were located in a freestream with velocity $V_{j} .{ }^{13,14,15}$ Based on the general trends in lift reduction with slipstream height shown by these researchers, Patterson and German suggest that the impacts of finite slipstream height can effectively be modeled as a simple reduction to the jet velocity. Specifically, for the cases of interest where the jet is caused by a propeller, a multiplicative factor, $\beta$, is applied to the propeller slipstream velocity, $V_{p}$, where $0 \leq \beta \leq 1$.

If the geometry shown in Figs. 3 and 4 is then modified so that $V_{p}$ is replaced by $\beta V_{p}$, the percentage increase in lift predicted by the point vortex model can be written as Eq. 2.

$$
\frac{\Delta L^{\prime}}{L_{\infty}^{\prime}}=\left(1-\frac{\beta V_{p} \sin i_{p}}{V_{\infty} \sin \alpha_{a}}\right) \frac{\sqrt{V_{\infty}^{2}+2 V_{\infty} \beta V_{p} \cos \left(\alpha_{a}+i_{p}\right)+\left(\beta V_{p}\right)^{2}}}{V_{\infty}}-1
$$


Although Patterson and German imply that a model for the $\beta$ parameter can be determined from the simulations of Ting et al., ${ }^{15}$ the precise variation of $\beta$ is unclear. Therefore, modeling to determine reasonable variations of the $\beta$ parameter were performed and are described in the following section.

\section{B. Quantifying Finite Slipstream Height Impacts}

To determine a model for the $\beta$ parameter, two-dimensional, inviscid simulations of a modified NACA 0012 airfoil ${ }^{\mathrm{c}}$ with an actuator disk upstream were performed in OVERFLOW, a structured overset grid CFD flow solver developed at NASA. ${ }^{16,17}$ The grid was designed so that there was a distance of 100 chord lengths between the airfoil and the outer domain. Each run was made by specifying a location of the actuator disk relative to the airfoil, the freestream velocity, the pressure increase across the actuator disk, and the angle of attack of the airfoil. The airfoil was pivoted about the quarter chord point, and the disk was placed perpendicular to the freestream velocity vector.

Due to the need for models that execute rapidly in the design process, a surrogate model for $\beta$ was developed from a series of OVERFLOW simulations. This surrogate model was developed as a function of three design parameters that are readily identifiable in early-phase design:

1. the ratio of the disk radius to the chord of the airfoil (i.e., $R / c$ ),

2. the velocity of the contracted slipstream far downstream of the disk (i.e., $V_{j}$ ), and

3. the distance upstream of the leading edge the disk is placed.

For this model, the vertical height of the disk and the angle of the disk relative to the freestream were kept constant.

Simulations were performed at a freestream Mach number of 0.2 with total Mach numbers far downstream of the actuator disk in the slipstream (i.e., $V_{j}$ ) of $0.25,0.3,0.4$, and 0.45 , which correspond to values of $V_{j} / V_{\infty}$ of $1.25,1.5,2$, and 2.25 , respectively. The ratio of the radius of the actuator disk to the chord length of the airfoil (i.e., $R / c$ ) was varied from 0.125 to 3.0. The distance the actuator disk was placed upstream of the wing leading edge, which is denoted here as $u$, was varied from values of $25 \%$ of the chord length up to three times the chord (or from $u / c=0.25$ to $u / c=3$ ). For the sake of brevity, only the results with a slipstream velocity ratio of $2^{\mathrm{d}}$ and selected results for how the lift augmentation varies with slipstream velocity are presented here. Additional details can be found in Ref. 18.

The results from the simulations are presented here in terms of the effective $\beta$ parameter implied by the output lift coefficient values. To determine $\beta$, an isolated airfoil is first run in OVERFLOW at the desired angle of attack. The airfoil is then run at the same angle of attack with an actuator disk upstream of it. The lift multiplier, $K_{L}$, is found as the ratio of the lift coefficient with blowing to the $c_{l}$ of the isolated airfoil. Since the slipstream is aligned with the freestream, the theory presented above indicates that the lift multiplier should be equal to the ratio of $V_{j}^{2} / V_{\infty}^{2}$ or $\left(V_{p}+V_{\infty}\right)^{2} / V_{\infty}^{2}$. However, the finite slipstream height (and other effects) may cause the lift to differ from this value. It is desired to find the apparent $V_{p}$ (i.e., $\beta V_{p}$ ) that can be used in the above theory to yield the calculated lift multiplier. This is done by calculating $\beta$ from Eq. 3.

$$
\beta=\frac{\sqrt{K_{L}}-1}{V_{p} / V_{\infty}}=\frac{\sqrt{K_{L}}-1}{V_{j} / V_{\infty}-1}
$$

The results of the OVERFLOW simulations for the $V_{j} / V_{\infty}=2$ case are presented in Fig. 6. ${ }^{\text {e }}$ This figure plots the $\beta$ value as a function of the ratio of radius of the actuator disk to the chord length of the airfoil $(R / c)$. Nine different $R / c$ values were evaluated: $0.125,0.25,0.5,0.75,1,1.5,2,2.5$, and 3 . This figure contains data from runs at four different $u / c$ distances - $0.25,0.5,1$, and 1.5 - as well as three angles of attack for each $R / c$ and $u / c$. The angles of attack of $1^{\circ}, 3^{\circ}$, and $5^{\circ}$ are denoted with asterisks, circles, and squares, respectively. The different line types and colors used in the figure refer to various $u / c$ values as shown in the legend.

Fig. 6 demonstrates that there is a strong influence on the lift generated from both the slipstream height and the distance upstream the disk is placed. Generally, increasing the distance of the actuator disk upstream increases the lift augmented by the airfoil (at a given $R / c$ ). This lift increase can be attributed to the increase

\footnotetext{
${ }^{c}$ i.e., one with zero trailing-edge thickness

di.e., $V_{j} / V_{\infty}=2$

e This speed was selected because it is on the higher-end of slipstream velocity ratios Borer et al. ${ }^{19}$ found to be practically obtainable from high-lift propellers at the speed and diameter ranges of interest here and to maintain parity with the calculations of Ting et al. ${ }^{15}$ who studied this velocity ratio.
} 


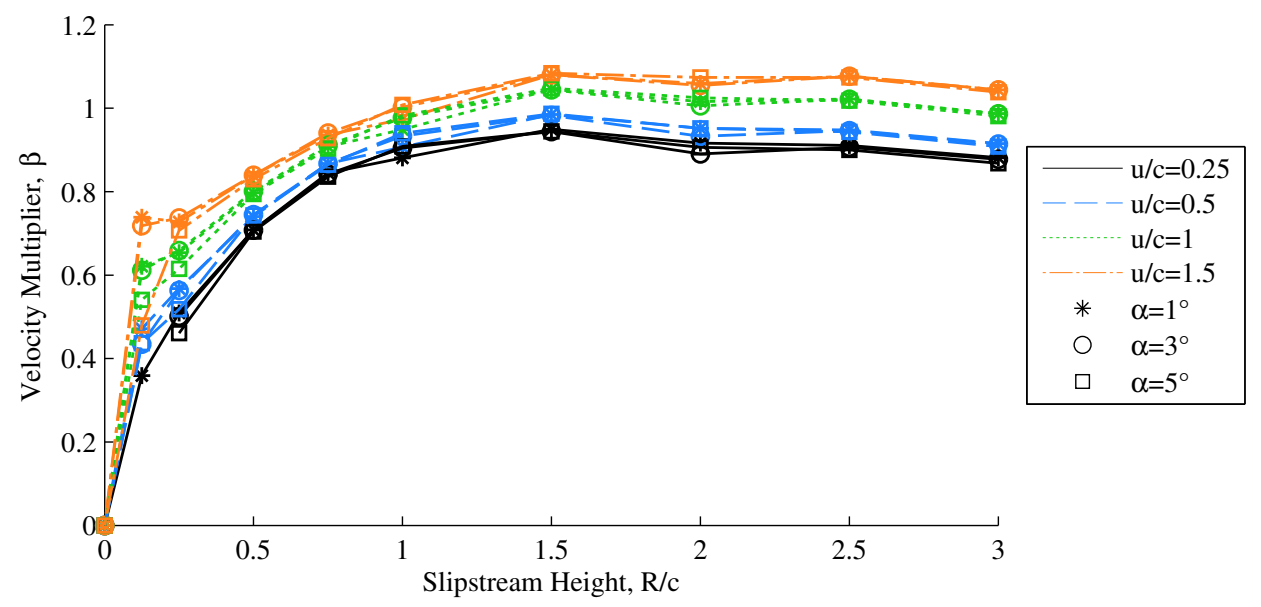

Figure 6. $\beta$ values as a function of slipstream height and upstream distance of the actuator disk determined from two-dimensional OVERFLOW simulations with the actuator creating $V_{j} / V_{\infty}=2$ far downstream at $1^{\circ}$, $3^{\circ}$, and $5^{\circ}$ angles of attack

in velocity that occurs as the slipstream contracts - greater distances upstream lead to increases in slipstream contraction and slipstream velocity.

A potentially vexing observation from Fig. 6 is that there are many cases where $\beta>1$. The theory presented by Patterson and German ${ }^{12}$ and the results from Ting et al. ${ }^{15}$ indicate that $\beta=1$ is an effective upper limit. However, these two theories are based on the assumption that the flow properties (i.e., density, temperature, and pressure) in the slipstream are unchanged from the freestream. In the OVERFLOW simulations, a static pressure increase is prescribed across the disk, which also results in changes in the temperature and density. These changes are more representative of what would actually occur in the flow aft of the propeller than the incompressible flow assumptions made in the previously cited studies. Incorporating the results from the OVERFLOW simulations into a model for $\beta$ that will be applied to the simple theory presented above will result in a model that will implicitly incorporate these flow property changes and, consequently, improve in accuracy.

Additionally, Fig. 6 indicates that differences in the lift multiplier between angles of attack are quite small with the exception of very low $R / c$ and high $u / c$ values. This observation helps justify the validity of the simple model presented above that is effectively agnostic to the angle of attack. Practically, this effective elimination of angle of attack as an independent variable is advantageous because it allows an accurate model to be built without including angle of attack as an input variable. The variations in $\beta$ at high $u / c$ and low $R / c$ values are the result of upper-surface blowing-like effects that occur when the slipstream is turned over the airfoil. Because these effects are limited to higher $u / c$ values than will likely be practical for high-lift propellers, these effects are not of particular interest for this present paper. ${ }^{f}$

Another general observation from Fig. 6 is that the lift augmentation from increasing slipstream heights is not a simple monotonically increasing function. The general decrease in lift for $R / c$ values above approximately 1.5 is caused by the effects of slipstream contraction/acceleration. For larger diameter actuator disks (i.e., larger $R / c$ values), the flow must travel a greater absolute distance downstream of the disk before it is fully accelerated than for a smaller disk. Therefore, if a small diameter actuator disk is placed at the same absolute distance from the airfoil as a large actuator disk, then the flow will have accelerated relatively more aft of the small disk than the large one. Since the curves are shown at constant values of the absolute distance upstream of the airfoil, there is a decrease in lift augmentation as $R / c$ becomes large.

The discussion thus far has focused on a single far downstream slipstream velocity (i.e., $V_{j} / V_{\infty}=2$ ), but the trends discussed above generally hold true for all other velocities studied. For the sake of brevity, not all the OVERFLOW simulation results will be presented in this paper, but this data can be found in Ref. 18 . To help visualize the variations in the effective $\beta$ parameter with the slipstream velocity, a reduced set of the data presented above is shown in Fig. 7 .

Fig. 7 plots the $\beta$ value obtained from the OVERFLOW simulations as a function of $V_{j} / V_{\infty}$ for three $u / c$

${ }^{\mathrm{f}}$ For a more thorough discussion of these effects, see Ref. 20. 


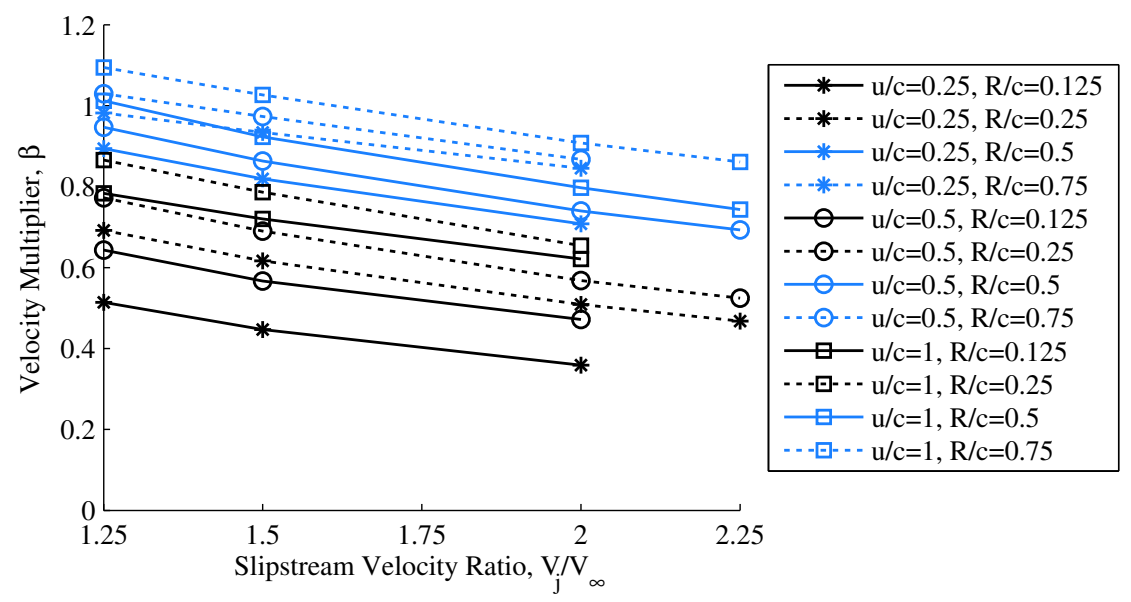

Figure 7. $\beta$ values as a function of slipstream velocity ratio $\left(V_{j} / V_{\infty}\right)$ for various actuator disk heights and upstream distances of the actuator disk determined from two-dimensional OVERFLOW simulations

values and four $R / c$ values. It is clear from this figure that there is a general decrease in the $\beta$ parameter as the slipstream velocity increases for the same disk height and upstream location. This decrease in $\beta$ implies that the lift augmentation becomes less effective the faster the slipstream velocity. Consequently, it may be more advantageous in certain situations to place the propeller farther upstream and/or use a larger diameter propeller than to attempt to increase the slipstream velocity by a large amount.

\section{Surrogate Model}

The data obtained from the OVERFLOW simulations and discussed above is useful for understanding the general trends that exist in the design space when varying the disk height and distance from the airfoil as well as the slipstream velocity. To make this data useful in a computational design environment, a surrogate model was fit to the OVERFLOW simulations results. This model represents changes in $\beta$ due to slipstream height as a fourth-order polynomial with coefficients that vary with $u / c$ and $V_{j} / V_{\infty}$. The model can be represented in a single equation as Eq. 4

$$
\beta=C_{0} X+C_{1} X\left(\frac{R}{c}\right)+C_{2} X\left(\frac{R}{c}\right)^{2}+C_{3} X\left(\frac{R}{c}\right)^{3}+C_{4} X\left(\frac{R}{c}\right)^{4}
$$

where $X=\left[\begin{array}{llllll}1 & u / c & (u / c)^{2} & (u / c)\left(V_{j} / V_{\infty}\right) & \left(V_{j} / V_{\infty}\right) & \left(V_{j} / V_{\infty}\right)^{2}\end{array}\right]^{T}$ and the $C_{i}$ values are coefficient row vectors of the form $C_{i}=\left[\begin{array}{llllll}c_{i, 0} & c_{i, 1} & c_{i, 2} & c_{i, 3} & c_{i, 4} & c_{i, 5}\end{array}\right]$. The resulting values of the coefficient row vectors $C_{0}, C_{1}, C_{2}, C_{3}$, and $C_{4}$ are given in Eq. 5, and these thirty numbers define the surrogate model.

$$
\begin{aligned}
C_{0} & =\left[\begin{array}{lllllll}
0.378269 & 0.748135 & -0.179986 & -0.056464 & -0.146746 & -0.015255
\end{array}\right] \\
C_{1} & =\left[\begin{array}{llllll}
3.071020 & -1.769885 & 0.436595 & 0.148643 & -0.989332 & 0.197940
\end{array}\right] \\
C_{2} & =\left[\begin{array}{llllll}
-2.827730 & 2.054064 & -0.467410 & -0.277325 & 0.698981 & -0.008226
\end{array}\right] \\
C_{3} & =\left[\begin{array}{llllll}
0.997936 & -0.916118 & 0.199829 & 0.157810 & -0.143368 & -0.057385
\end{array}\right] \\
C_{4} & =\left[\begin{array}{llllll}
-0.127645 & 0.135543 & -0.028919 & -0.026546 & 0.010470 & 0.012221
\end{array}\right]
\end{aligned}
$$

The three-dimensional input of the surrogate model makes visualization of the model output somewhat difficult. However, the general trends that result from changes in $R / c$ or $u / c$ can be readily observed from the raw data in Fig. 6, and the general variation with $V_{j} / V_{\infty}$ can be seen in Fig. 7 .

To ascertain the accuracy of the surrogate model, the model was evaluated at the $R / c, u / c$, and $V_{j} / V_{\infty}$ points used to fit the model as well as at other validation points. In evaluating all the model fit points and 
validation cases the surrogate model has an $R^{2}$ value of 0.9858 compared to an $R^{2}$ of 0.9856 on simply the model fit points. If the $R / c=0.125$ values are excluded, these $R^{2}$ values are 0.9917 and 0.9914 , respectively. Analysis of the residuals - i.e., the difference between the model prediction and the actual values from the OVERFLOW simulations - also provides insight into the accuracy of the model. Fig. 8 shows two ways of visualizing the residuals data: Fig. 8(a) plots the residuals versus the model predicted values and Fig. 8(b) is a histogram of the residuals. The observed relatively even scatter of the points around a mean of zero is

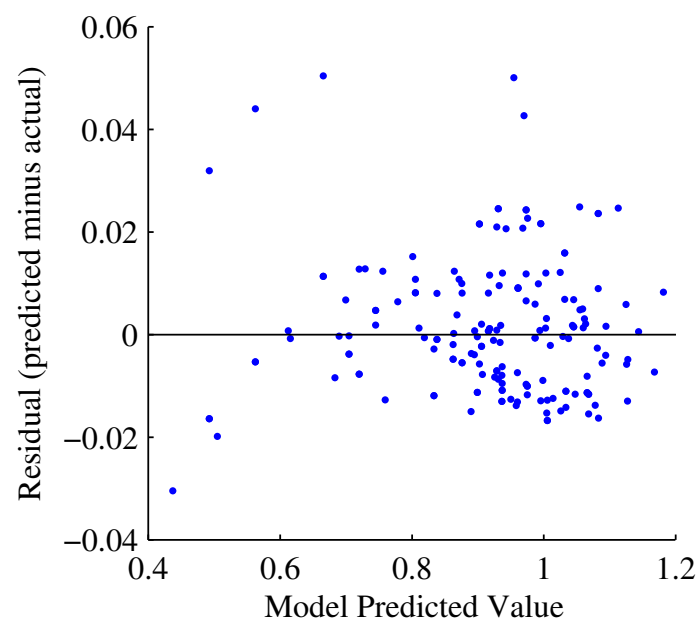

(a) Residual values vs model predicted values

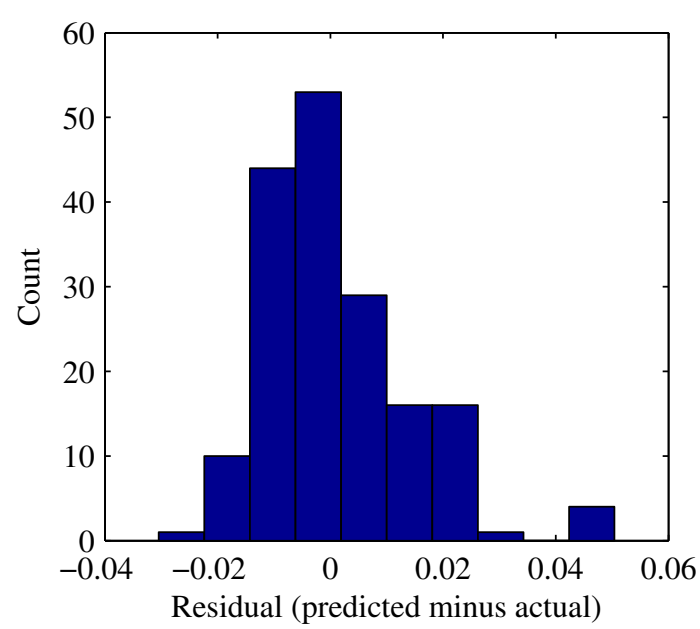

(b) Residuals histogram

Figure 8. Residual error of surrogate model

desirable and indicative that the surrogate model provides a reasonable fit to the data. The mean of the residuals is 0.001 with a standard deviation of 0.0134 . These low values are an additional indication that the model provides a good fit to the data.

The data shown in Fig. 8 also provide insight into where the model is least accurate. Specifically, the model deviates most from the OVERFLOW simulations at very low values of $R / c$. Additionally, larger deviations are observed at very high $V_{j} / V_{\infty}$ values (i.e., at 2.25). Consequently, caution should be exercised if the model is desired to be used for cases with very small propellers or very large slipstream velocity increases.

\section{Design Space Exploration to Select the Number of High-Lift Propellers}

In this section, the analysis performed to select the number of propellers for the SCEPTOR aircraft will be explained. A design space exploration procedure is followed to provide insights about the design space in addition to determining an overall configuration design. In trying to determine a desired number of propellers, there are many metrics of interest to a designer of high-lift propeller systems. These metrics include the total power required, total thrust generated, individual motor torque/power required, performance after a critical motor or propeller failure (which, for example, may cause a yawing moment and a loss of lift), total system weight (including motors, controllers, nacelles, wiring, etc.), nacelle (cruise) drag, reliability, cost, and complexity.

Prior to discussing the design space exploration procedure, a brief comment on the thrust from highlift propellers is appropriate. Aircraft with high-lift propellers will likely be designed with relatively small wings that require blowing from the high-lift props to meet low-speed performance requirements. These props will inherently produce thrust in low-speed conditions, and it is possible that the thrust generated to provide sufficient lift will make it impossible for the aircraft to descend at the desired speed. Consequently, the thrust generated by high-lift propellers should likely be the minimum possible to provide the required lift augmentation. Consequently in the discussions that follow, it is assumed that the thrust should be minimized. 


\section{A. Design Assumptions and SCEPTOR Aircraft Information}

In designing the SCEPTOR aircraft, the team first selected initial wing and cruise propeller designs (i.e., the propellers that provide cruise thrust and are located at the wing tips) under the assumption that a high-lift propeller system could be designed to provide sufficient lift at low speeds. In practice, an approach similar to the following could be implemented in parallel with the wing design process to obtain a more advantageous overall aircraft design.

The details of the overall configuration and the design process followed to develop this configuration will be published in a companion AIAA Aviation 2016 paper by Borer et al. ${ }^{21}$ For the purposes of the present study, the following geometric parameters are all that are required to be known:

- the trapezoidal wing has a span of $31.6 \mathrm{ft}$, an average chord of $2.11 \mathrm{ft}$, and a taper ratio of 0.7 (as defined from the wing root at the aircraft centerline);

- the wing has $2^{\circ}$ of washout and the root of the wing is inclined upward at $2^{\circ}$ (so that at cruise the aircraft should fly at approximately a $0^{\circ}$ angle of attack);

- the fuselage spans $3.95 \mathrm{ft}$ of the wing;

- the wing is flapped beginning just outside the fuselage junction and moving out $10.4 \mathrm{ft}$ along the span;

- the wing is made of a custom airfoil and single slotted flap, which together exhibit a zero-lift angle of attack of approximately $-20^{\circ}$ and a maximum sectional lift coefficient of approximately 2.75 at a $10^{\circ}$ angle of attack; and

- the cruise (wingtip) propeller diameter is $5 \mathrm{ft}$.

Additionally, the aircraft is being designed to achieve a 55 knot stall speed (at standard sea level conditions) and will have a gross weight of 3,000 lb. Finally, we will assume that the aircraft's high-lift nacelles will be oriented so that when the aircraft is at a $0^{\circ}$ angle of attack, the nacelles are at a $0^{\circ}$ inclination relative to the aircraft reference frame. ${ }^{\mathrm{g}}$

To put practical limits on the design space for the high-lift propeller system for the SCEPTOR aircraft, the following assumptions were made:

- All high-lift propellers have the same design (i.e., the same diameter, chord distribution, twist distribution, etc.), and the prop blades use a single airfoil, the MH114. ${ }^{22}$

- The configuration has an equal number of high-lift props on either side of the fuselage.

- The high-lift propellers fill the entire span of the wing from the edge of the fuselage to the edge of the wingtip propellers without overlapping any of the propellers (in order to reduce the acoustic signature produced by interacting prop tip vortices).

- Propeller tip speeds at the design condition are set to $450 \mathrm{ft} / \mathrm{sec}$, which is about half the typical tip speed of similarly-sized aircraft, to reduce noise.

- The propellers are placed relatively close to the wing chord line so that the surrogate model developed above will provide accurate results.

- The unblown maximum lift coefficient of the wing (with flaps) is 2.6.

- The appropriate design point for the high-lift propellers is the desired blown stall speed.

- The propellers should have an odd number of blades to reduce the acoustic signature.

In order to obtain reasonable estimates of the motor weight and diameter, simple models were developed based on the Joby JM1 motor, ${ }^{23}$ which is a candidate motor for the SCEPTOR flight demonstrator. Because the torque and power levels of the JM1 are likely not sufficient for all the high-lift propeller systems under consideration, a "rubberized" JM1 model is developed.

Because the design of electric motors is beyond the scope of the present work, it is assumed here that, as the power requirements of the high-lift propellers increase, the diameter of the motor (and thus the nacelle size) will also increase. Such an approach should capture the general trends of increased power requirements and allows for a simple model to be developed whose only input is the required power level. ${ }^{\mathrm{h}}$ Based on the specifications of the Joby motors, a specific power of 2 horsepower per pound ${ }^{\mathrm{i}}$ and a specific diameter of 1.1 inch per pound were selected. To determine the motor weight, the power required at the sizing condition is divided by the specific power. Similarly, the diameter of the motor is determined by multiplying

\footnotetext{
${ }^{g}$ This implies that a nacelle placed directly at the wing root would be angled downward relative to the wing chord by $2^{\circ}$ and a nacelle placed directly at the tip would be directly aligned with the chord line.

${ }^{\mathrm{h}}$ In general, electric motors can be made of varying weights and diameters to achieve the same torque or power. For example, a smaller diameter motor can provide the same torque or power as a larger diameter motor, but generally the larger diameter motor will be lighter.

${ }^{\mathrm{i}}$ The specific power assumed here is slightly lower than the Joby Motor data would suggest to account for the additional weight of a controller. For simplicity of explanation, this total weight is referred to as the motor weight.
} 
the estimated motor weight by the specific diameter. To avoid potentially unrealistic results, limits on the maximum and minimum motor diameters were set to 18 inches and 3 inches, respectively. ${ }^{j}$

The motor diameter affects the propeller design and the nacelle drag. Propeller blades cannot be designed for radial stations less than the motor radius, and larger motor diameters will reduce the effective disk area, which will impact the power and/or thrust of the prop. Additionally, the motor diameter will effectively set the nacelle size, which will directly impact the drag of the configuration. For the studies presented here, Raymer's ${ }^{24}$ component drag buildup method - a simple handbook method based on skin friction coefficients, form factors, wetted areas, and interference factors - is used for estimating the nacelle drag. The nacelle is modeled in OpenVSP ${ }^{25,26}$ with a pod component assuming a fineness ratio ${ }^{\mathrm{k}}$ of 6 to obtain the wetted area. Raymer's suggested interference factor (1.3) and form factor equation for nacelles are employed to estimate the drag of the configuration at cruise.

\section{B. Design Space Exploration Process}

With a given wing design and the above assumptions, the high-lift propeller system can be defined by selecting the number of props (which implies a prop diameter), a blade shape (i.e., twist and chord distributions), and the number of blades. To select these parameters, an exploratory design method is followed-i.e., many candidate designs are created and evaluated, then a "preferred" design is selected. This exploratory method is instructive for showing the tradeoffs that exist in the design of high-lift propeller systems as will be shown below.

The overall exploratory design procedure consists of the following steps:

1. Select the number of high-lift props

2. Estimate the motor/hub diameter

3. Design many props (by varying the design section lift coefficient) that provide the required induced velocity to produce the desired blown stall speed

4. Analyze all props at off-design conditions

5. Select the most-preferred prop design for this particular number of props

6. Repeat this procedure (back to step 1) for all numbers of props

7. Compare the most-preferred design for each number of props, and select the number of props with the most-preferred overall performance

The following sub-sections describe these steps in more detail.

\section{Designing Propellers}

Two separate propeller design methods are studied here: one traditional minimum induced loss (MIL) design method and the high-lift propeller design method presented in Ref. 20. The propellers are designed for the desired 55 knot blown stall speed condition. The required lift augmentation for level flight at 55 knots taken with the geometry of the wing provide a value of the required average axial velocity that must be produced by each high-lift propeller. This required axial velocity is used as an input to the propeller design process.

Additionally, both propeller design methods require inputs for the blade element airfoil properties. Specifically, lift and drag estimates of the airfoil sections as well as a design section lift coefficient are required. To select an appropriate section lift coefficient, a sweep of 40 different design lift coefficient values ranging from 0.1 to 1.77 (the approximate stall $c_{l}$ ) is performed. For each of these design lift coefficient values, a new propeller design is generated, and these designs are evaluated at off-design conditions, as described below, before selecting the desired section lift coefficient.

The traditional propeller designs are generated with the open-source propeller analysis and design tool XROTOR, ${ }^{27}$ which is based on the MIL theory of Larrabee. ${ }^{28,29}$ To design a minimum induced loss rotor, XROTOR requires inputs for the desired thrust from the propeller and blade element section properties in addition to freestream flow conditions. An iterative procedure is performed to determine the appropriate input thrust value to XROTOR to produce the needed average axial velocity. The initial guess of the thrust

\footnotetext{
${ }^{j}$ Motor sizes could potentially be smaller than the minimum, but the nacelle must have sufficient volume to hold the motor and likely the controller as well. Additionally, motor sizes may realistically become larger, but this would come with a change in motor architecture, which would likely increase the specific power. This maximum limit is not reached in any of the design studies performed below.

${ }^{\mathrm{k}}$ The fineness ratio is defined as the ratio of the length to the maximum diameter. Currently the fineness ratio in OpenVSP's pod component uses the radius in its calculation instead of the diameter. This error is accounted for in the modeling presented here.
} 
is determined from simple momentum theory. If this thrust estimate does not produce the correct average axial velocity, the thrust input to XROTOR is varied until the desired average induced axial velocity is produced. It is possible that such an iterative procedure may never converge because the required thrust is simply too high for the other input parameters. In these cases, no feasible solution can be found with the MIL design method.

Specific high-lift propeller designs are generated for each number of props and design $c_{l}$ following the method outlined in Ref. 20. It is possible that this design process will be unable to design a propeller in certain situations, but in general the method can provide designs for many different inputs. This robustness is both a strength and a weakness of the method. If left unchecked, the designs generated can have unrealistically large chord lengths in an attempt to generate the desired forces. To ensure that reasonable designs resulted during the design process, the chord length of all designs generated with the simple high-lift prop design method are limited to have chord lengths of no more than $40 \%$ of the radius. If this limit is reached, some additional iteration of the method is required to obtain propeller designs that achieve the desired average induced axial velocity.

\section{Off-Design Evaluation}

After each propeller is designed, it is also evaluated at off-design conditions of 30 and 90 knots to ensure reasonable performance exists in these off-nominal cases. A velocity of 30 knots approximates the conditions that would be present over much of a takeoff ground roll, and flight at 90 knots is likely close to an upper-limit on the speed range in which the high-lift propellers will ever be practically operated for the SCEPTOR aircraft. ${ }^{1}$ By analyzing the propellers at these two off-nominal conditions, the general variations in performance over a range of practical operating speeds can be assessed to ensure that the designs provide acceptable performance.

\section{Selecting the Most-Preferred Propeller Design for a Given Number of Propellers}

In the design exploration process, many propeller designs are generated for a given number of blades, number of propellers, and prop design method by varying the design lift coefficient. If only point-design characteristics are of interest, then the "optimum" design $c_{l}$ will likely be the lift coefficient for maximum lift-to-drag ratio. However, when off-design conditions are evaluated, the best design $c_{l}$ may be substantially different than the $c_{l}$ for maximum $L / D$. Because the high-lift propellers are fixed pitch, as the freestream velocity is changed the local angle of attack of the blade sections will vary (for a constant RPM). This change in local angle of attack can lead to stalled blade sections and/or reduced performance at off-design conditions.

For the purposes of the design exploration process presented here, the performance of many propellers designed with different design section lift coefficients is visualized, and general trends in performance are observed. Based on the observed trends, a preferred propeller is selected based on a simple rule that is described below.

To visually explore the changes in performance as the design section lift coefficient is varied, the change in six metrics of interest are plotted as a function of the design $c_{l}$ in Fig. 9. These metrics are the thrust, power, torque, number of stalled airfoil sections, average swirl angle, and average induced axial velocity, which are plotted in Figs. 9(a) through 9(f), respectively. The variations of these metrics with the design $c_{l}$ at the design point of 55 knots standard sea level conditions are denoted by black asterisks. The off-design performance characteristics at velocities of 30 knots and 90 knots are shown with gray circles and triangles, respectively.

The information in Fig. 9 applies to a case where five-bladed propellers were designed with a minimum induced loss method for a version of the SCEPTOR aircraft that has twelve high-lift props. Forty separate propellers were designed with design lift coefficients ranging from 0.1 to 1.77 , and each point in the figure refers to one of these propellers. Although this figure applies to only one specific case, the trends observed here generally apply to all other numbers of props and blades when sweeping over the design $c_{l}$.

Each of the propellers is designed to produce the same average induced axial velocity at the design condition as shown in Fig. 9(f). Interestingly, the thrust, power, torque, and swirl are virtually constant for all propellers at the design condition with the exception of props designed with low design lift coefficients (i.e., less than approximately 0.5 ). This is a testament to the intuition that momentum theory can help build

\footnotetext{
${ }^{1}$ The wing is capable of generating more than sufficient lift without the high-lift propellers at this higher-speed condition.
} 


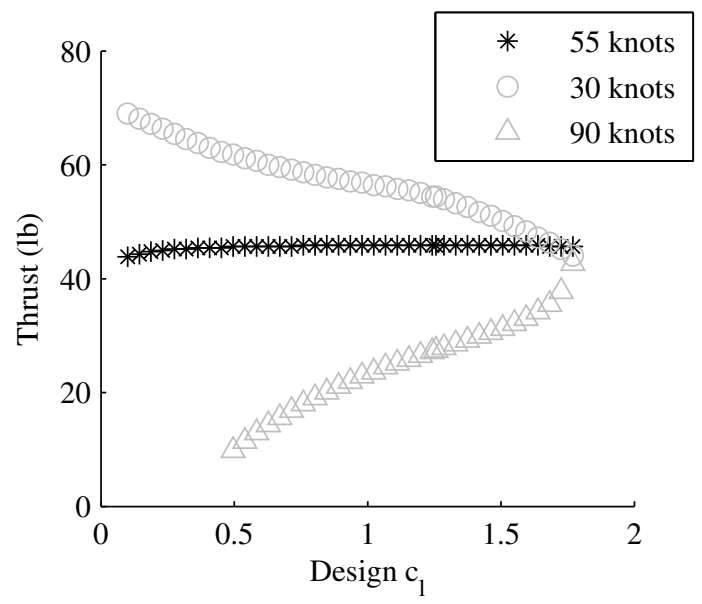

(a) Thrust

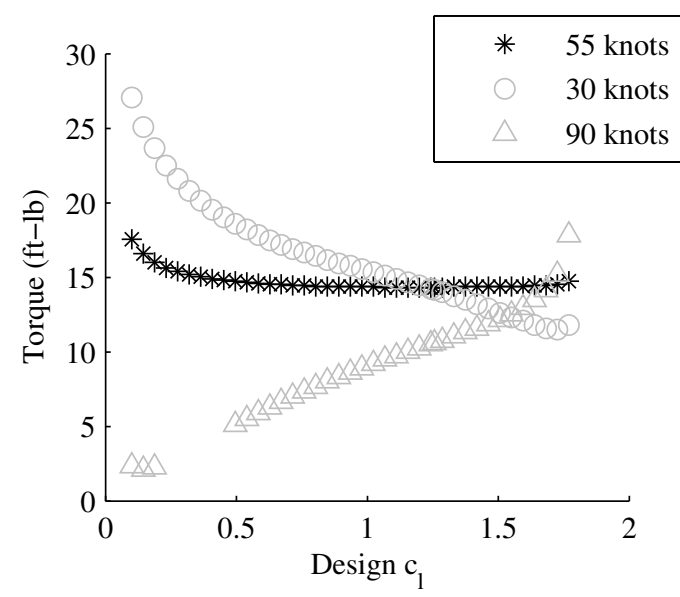

(c) Torque

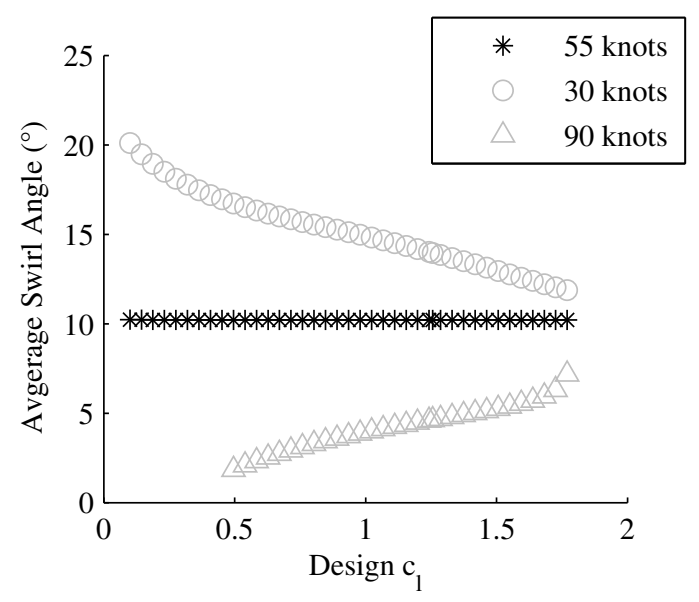

(e) Average swirl angle

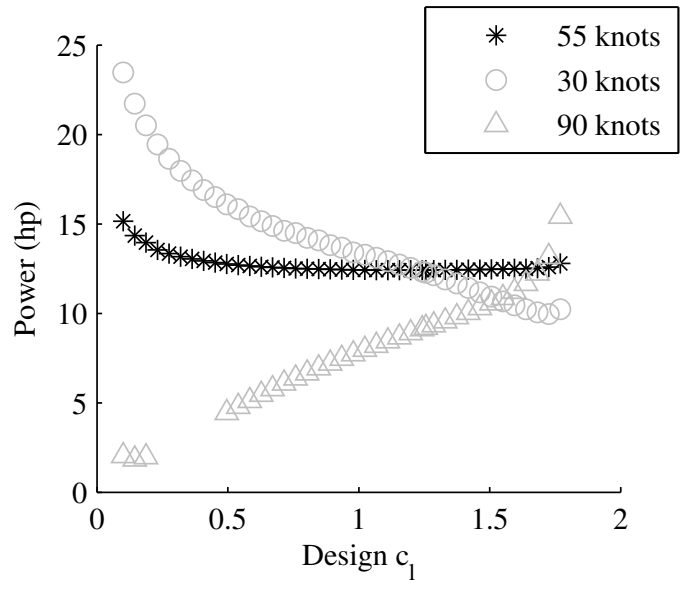

(b) Power

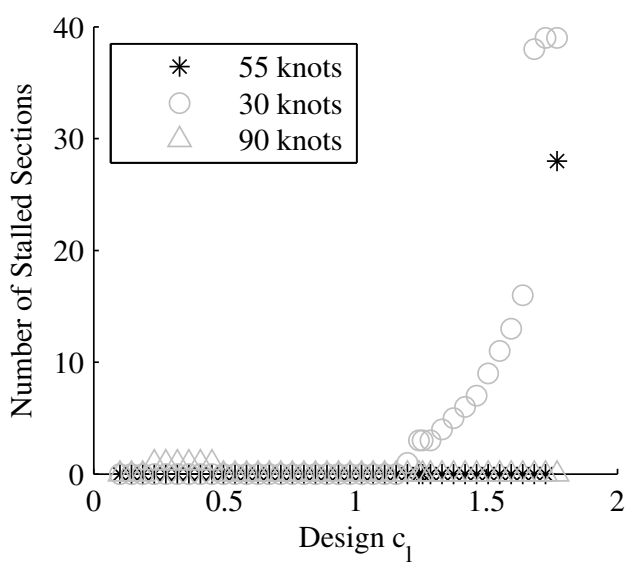

(d) Number of stalled airfoil sections (where the blade is designed with 40 sections)

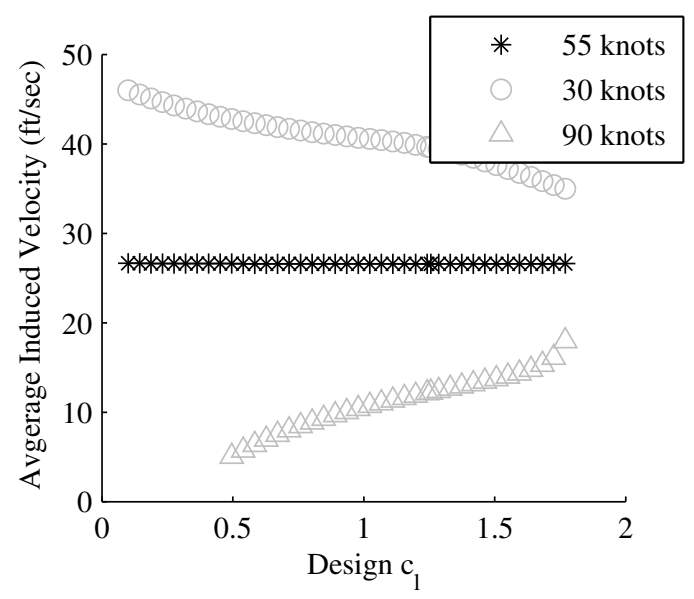

(f) Average induced axial velocity

Figure 9. Comparison of six metrics of interest when varying the design $c_{l}$ of a five-bladed propeller in a minimum induced loss design method for a twelve propeller configuration on the SCEPTOR aircraft 
(though momentum theory under-predicts the thrust and power required to produce this desired average induced axial velocity).

In selecting the most preferred propeller design, both the on- and off-design performance of the prop are considered. An ideal high-lift propeller would produce the desired average induced axial velocity with very low thrust, power, torque, and swirl while not experiencing stall over any blade sections (to reduce the acoustic signature of the props) at all flight conditions. Additionally, it may be advantageous for the propeller performance to show relatively little variation from on-design to off-design conditions. Based on these desired characteristics, high design lift coefficients can be eliminated because they lead to stalled blade sections at low-speed operations. Similarly, low design $c_{l}$ values lead to increased torque and power requirements. Furthermore, Fig. 9 indicates that the least variation between on- and off-design conditions occurs at moderate to high design $c_{l}$ values. With these trends in mind, the most-preferred design $c_{l}$ in the design space exploration process is selected as the highest design $c_{l}$ that does not cause stall over any blade sections at any of the conditions evaluated. For the case shown in Fig. 9, a design $c_{l}$ of 1.15 is selected. All other cases (i.e., number of high-lift propellers and number of blades) are evaluated in the same manner, and the most-preferred propeller design is determined for all of these conditions.

\section{Selecting the Number of Propellers}

Once the most-preferred high-lift propeller design is determined for each number of props, these designs are then compared to one another to select the most advantageous number of propellers. A design space visualization procedure similar to that performed to determine the design $c_{l}$ is followed here to provide insight into the trades that exist with high-lift propeller systems.

In selecting the number of propellers, the aircraft's performance under off-nominal circumstances must be considered in addition to on-design performance because this performance may change the resulting aircraft design. For example, in the design of a conventional twin-engine general aviation aircraft, the aircraft's performance with the critical engine inoperative can be a driving factor in the design of the empennage and/or power requirements of an engine. In the case of high-lift propellers, the most critical single-point failure corresponds to the high-lift propeller that ultimately generates the most lift. Interestingly, this "critical high-lift motor" is likely the inner-most motor, since it will drive a propeller that typically blows the largest area of the wing and has a flap. ${ }^{\mathrm{m}}$ Additionally, there could be a second critical motor in relation to the yawing moment that is more similar to the conventional "critical engine." If a reasonably high thrust is required from the high-lift propellers, the relatively large spanwise distance at which high-lift motors may be placed could result in a large yawing moment in the event of a motor failure. This "critical yawing motor" is the one that generates the most yawing moment, which under many circumstances will be the farthest outboard motor. The yawing moment with the critical yawing motor inoperative and the change in stall speed with the critical high-lift motor inoperative are both tracked in the following design space exploration.

The results from the design space exploration for the SCEPTOR aircraft are shown in Figs. 10 and 11. To provide insight into the design space, ten separate metrics are tracked for each design. ${ }^{\mathrm{n}}$ These metrics are

1. the total thrust of all high-lift propellers (Fig. 10(a)),

2. the total power required from all high-lift motors (Fig. 10(b)),

3. the required torque for each propeller (Fig. 10(c)),

4. the required power for each prop (Fig. 10(d)),

5. the average swirl angle (Fig. 10(e)),

6. the total motor weight (Fig. 11(a)),

7. the nacelle parasite drag estimate at cruise (Fig. 11(b)),

8. the yawing moment that would result from the loss of the critical yawing motor (Fig. 11(c)),

9. the total yawing moment generated by all propellers on one side of the aircraft (Fig. 11(d)), and

10. the stall speed resulting from the loss of the critical high-lift motor (Fig. 11(e)).

In each of the sub-figures, six different propeller designs are shown for each number of high-lift propellers. These six designs correspond to 3-bladed, 5 -bladed, or 7-bladed propellers that were designed with one of the two different propeller design methods. The minimum induced loss designs are shown with black markers

\footnotetext{
${ }^{m}$ It is certainly possible other motors could be the critical high-lift motor. For example if the inner-most high-lift propeller blows a section of wing without a flap while other props blow flaps.

${ }^{\mathrm{n}}$ These metrics are all evaluated at the design condition of 55 knots standard sea level conditions unless otherwise noted.
} 
and denoted with 'MIL' while those designed with the high-lift propeller design method presented in Ref. 20 are shown in blue and denoted by 'HLP.'

One may notice that for each number of high-lift propellers not all design methods and/or number of propellers are represented in the figures. For example, for 14 and more propellers, there is no MIL, 3-blade design shown. The lack of a marker indicates that no propeller was designed successfully for these conditions that meets the constraints mentioned above in Section 3. As the number of propellers is increased, generally fewer combinations of design method and number of blades generate feasible designs.

When studying Figs. 10 and 11, the many competing tradeoffs inherent in the design of high-lift propeller systems become apparent. Certain metrics (e.g., swirl) indicate that the fewest number of high-lift propellers should be selected; other metrics (e.g., stall speed with one motor inoperative) indicate that the highest number of props is most advantageous; other metrics (e.g., total power) suggest that some number of props between the extremes is best. One could justify selecting almost any number of propellers based on this information!

Additionally, although many metrics have clear directions of optimality (e.g., a minimum power propeller is desired), other metrics may not have a clear "optimal" direction, which makes the selection of an appropriate number of propellers even more difficult. One example metric without a clearly optimal direction is the yawing moment per side, which is shown in Fig. 11(d). It may be advantageous for the high-lift propellers to be capable of generating a large yawing moment to counteract the adverse yawing moment that would arise if a cruise propeller failed. However, if there were to be a failure of all high-lift propellers on one side of the wing, ${ }^{\circ}$ it would be more advantageous if a small yawing moment resulted. To provide a reference for the relative magnitudes of the yawing moments shown in Figs. 11(c) and 11(d), an estimate of the one engine out yawing moment that the Tecnam P2006 $\mathrm{T}^{\mathrm{P}}$ would generate at 55 knots and takeoff power is shown with a dotted line in Fig. 11(d). ${ }^{\mathrm{q}}$ It appears that most MIL designs would generate approximately the same yawing moment as the base Tecnam while most HLP designs would generate less moment in this case. This indicates that the tail of the Tecnam is appropriately sized to handle the worst-case yawing moment from the high-lift props. However, if it is desired that these high-lift props counteract the yawing moment from one of SCEPTOR's wingtip cruise props, then they will be woefully inadequate since the wingtip propellers will generate substantially larger yawing moments than the baseline Tecnam propellers because they are placed further outboard. ${ }^{\mathrm{r}}$

Another off-nominal situation of note is the change in the stall speed if the critical high-lift motor fails, which is shown in Fig. 11(e). Because the high-lift propellers are all designed to provide the same lift augmentation, the loss of lift when a motor fails is equivalent ${ }^{\mathrm{s}}$ between all propeller designs at a set number of propellers. Configurations with fewer high-lift props will experience greater changes in lift, but the likelihood of a motor failing is reduced. ${ }^{\mathrm{t}}$

It is also interesting to note from Figs. 10 and 11 that the optimum number of propellers for many metrics changes based on the propeller design method employed. For example, Fig. 10(b) indicates that the minimum total power is obtained with 12 high-lift props when using the MIL method while 14 props provides the lowest power solution from the HLP method. Similarly, the number of blades can also impact the optimum number of propellers for certain metrics - particularly for the MIL design method. For example, the minimum motor weight occurs with 12 motors for the MIL 5-bladed designs, but the MIL 7-bladed designs suggest 14 motors leads to the lowest motor weight as shown in Fig. 11(a). These observations suggest that the propeller design method employed and the number of blades selected can have significant impacts on the performance characteristics of the high-lift prop system.

Ultimately, a decision on the number of high-lift propellers (and likely the propeller design method and number of blades) must be made. Ideally the number of propellers with the lowest power would also have the lowest total motor weight, nacelle drag, and induced swirl as well as the smallest change in stall speed if the critical motor is lost. Unfortunately, this is not the case. To further complicate matters, there are other factors to consider when selecting the number of high-lift propellers that may not be easily quantified in the

\footnotetext{
${ }^{\circ}$ a scenario that should be very low probability with a proper electrical system architecture

pRecall that the SCEPTOR aircraft is a modified version of the P2006T. The empennage of the SCEPTOR aircraft will be unchanged from the baseline aircraft.

${ }^{\mathrm{q}}$ This is a simple calculation assuming a $67.5 \%$ propeller efficiency and the engine operating at full power of $73.5 \mathrm{~kW}$.

${ }^{\mathrm{r}}$ A rough estimate is that one of the wingtip propellers at the 55 knot condition will generate a yawing moment of approximately $5,275 \mathrm{ft}-\mathrm{lb}$ if operated at full power.

s based on the models employed here; in reality there will be small changes between propeller designs

${ }^{\mathrm{t}}$ assuming the motors and propellers have equivalent reliabilities
} 


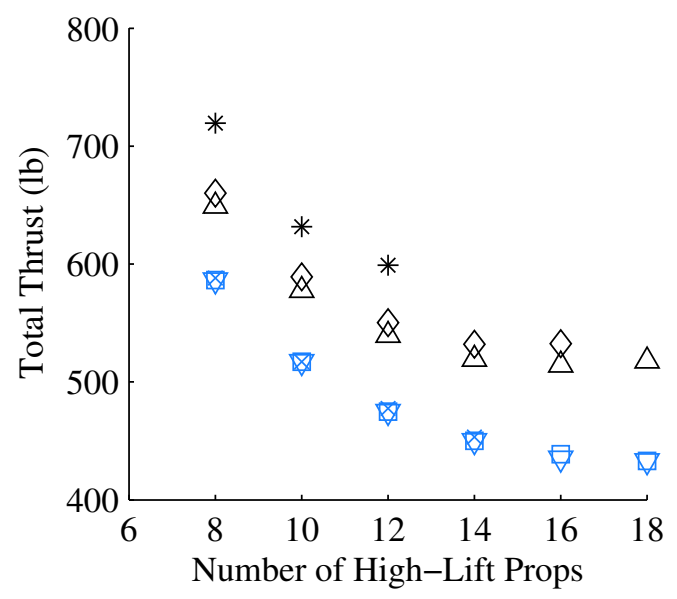

(a) Total thrust

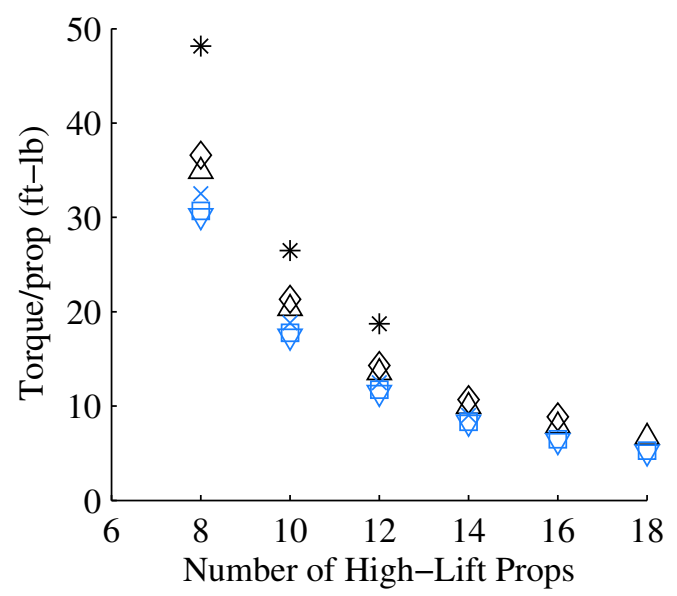

(c) Torque per prop

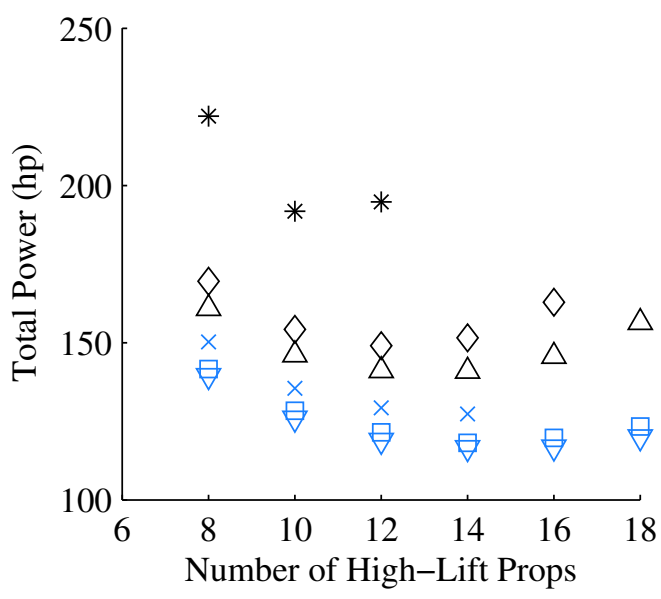

(b) Total power

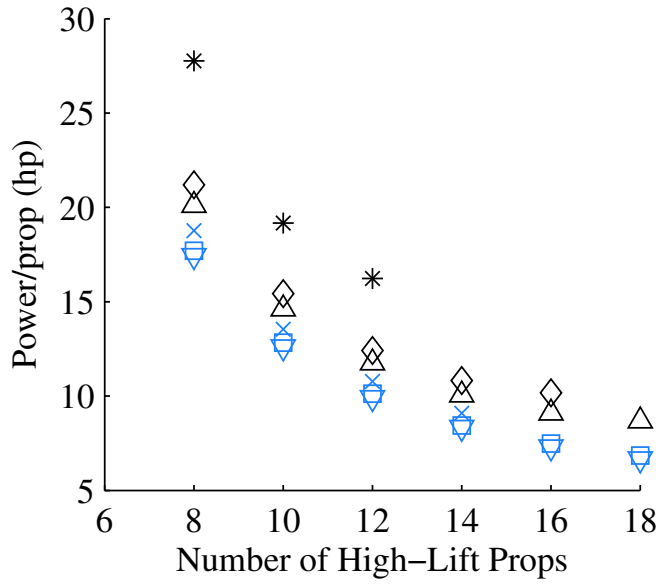

(d) Power per prop

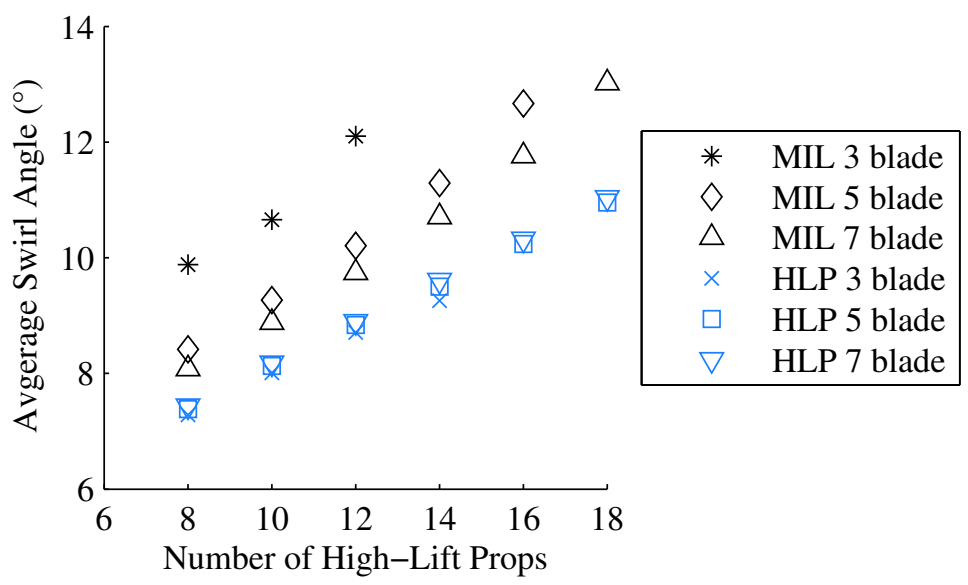

(e) Average swirl angle

Figure 10. Part 1 of 2: Comparison of metrics of interest when varying the number of propellers and the number of blades per propeller for conventional and high-lift prop design methods 


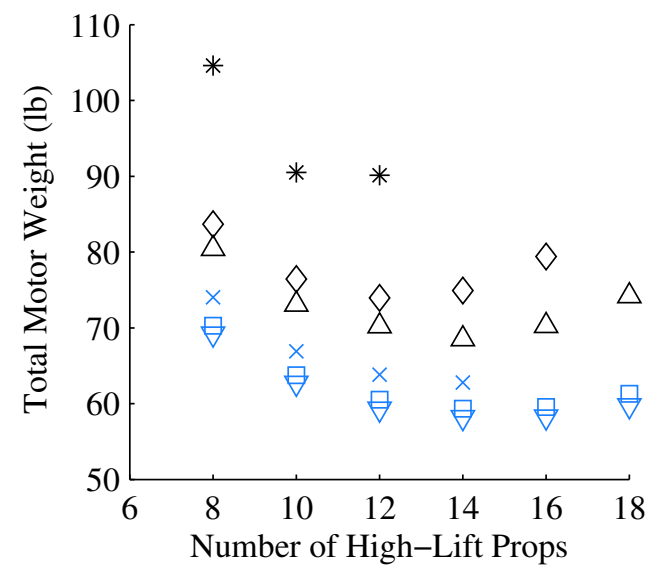

(a) Motor weight

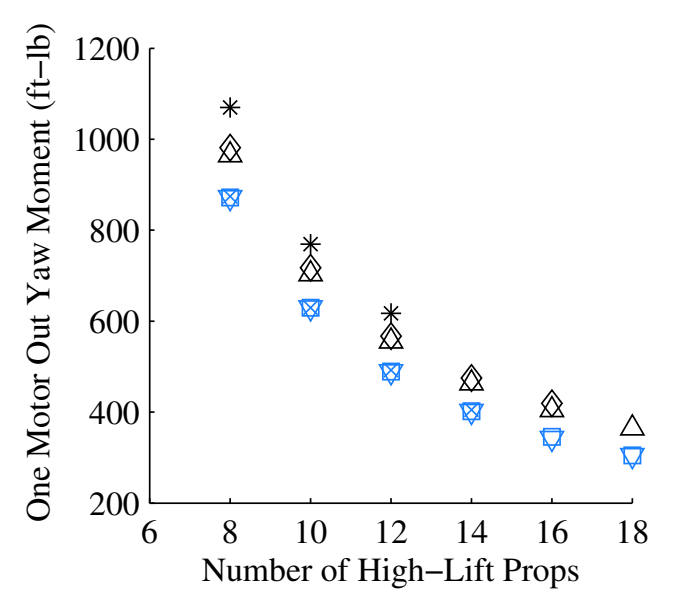

(c) Yawing moment with critical motor inoperative

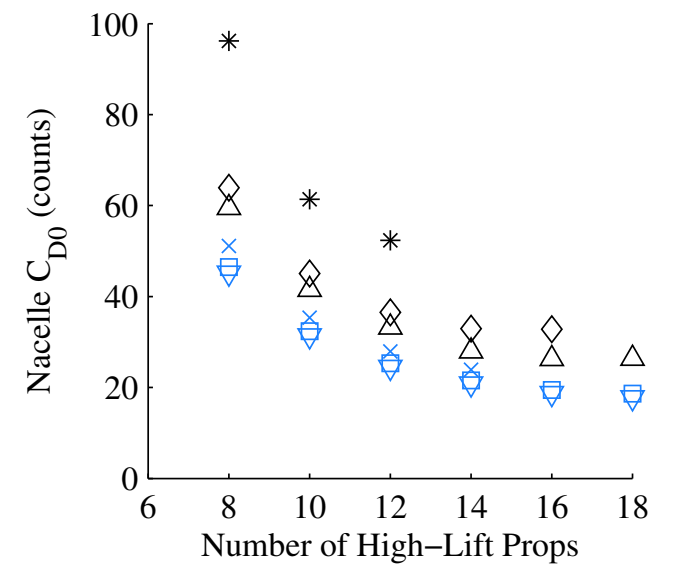

(b) Nacelle parasite drag at cruise (150 knots, 8,000

$\mathrm{ft}$ )

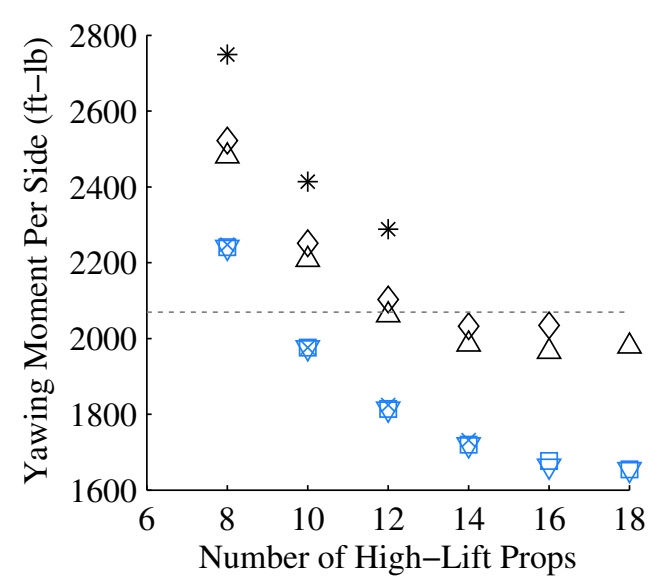

(d) Yawing moment of all motors on one side. (The dotted line is an estimate of the Tecnam P2006T yawing moment with one engine out.)

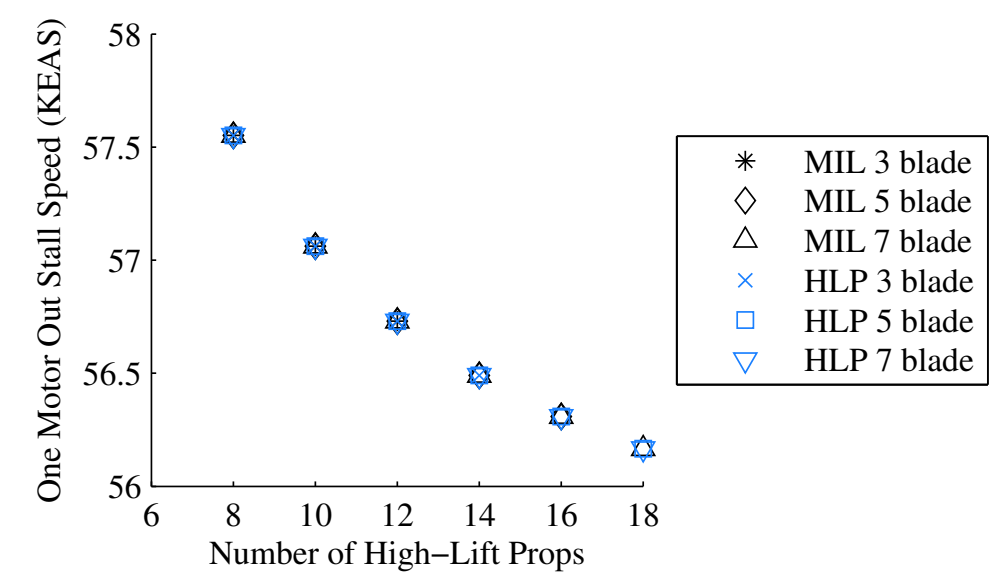

(e) Stall speed with critical motor inoperative

Figure 11. Part 2 of 2: Comparison of metrics of interest when varying the number of propellers and the number of blades per propeller for conventional and high-lift prop design methods 
early conceptual design phase such as

- complexity (e.g., additional motors require more parts),

- cost (including acquisition, maintenance, and disposal costs),

- reliability (e.g, the more motors/propellers the system has, generally the higher the probability of failure of any single component, but the lower the consequences if there is a failure),

- availability of commercial off-the-shelf components (e.g., an electric motor with the proper torque/power requirements) for use in the design,

and so forth.

Overall, it seems that either 12 or 14 high-lift propellers are likely the most advantageous number of propellers to select in this case. Depending on the prop design method and number of blades, either 12 or 14 propellers provides the lowest power; additionally, the total thrust and nacelle drag values for 12 and 14 props are close to the lowest values predicted. Finally, 12 and 14 propellers provide a "happy medium" in terms of average swirl angle, resulting stall speed with the critical high-lift motor inoperative, and complexity. Earlier design studies only considered minimum induced loss propeller designs; consequently, 12 propellers were selected for the final SCEPTOR flight demonstrator aircraft because this configuration was estimated to require the lowest power as shown in Fig. 10(b). Minimizing the power is critical for the aircraft due to the limited energy available in the batteries.

\section{Conclusions}

An improvement to the design method of Patterson and German to quickly estimate the lift augmentation of high-lift propellers on downstream wings has been presented, and the improved model was utilized to help determine the configuration design for the SCEPTOR aircraft. The improvement abstracts data from twodimensional, inviscid CFD simulations into a surrogate model that accounts for the effects of finite slipstream heights, propeller upstream distances from the wing, and slipstream velocities. This surrogate model enables these corrections to efficiently be incorporated into the conceptual design process. Additionally, the use of this surrogate model effectively removes the incompressible flow assumptions of the original method.

The design space exploration for the high-lift propeller system of the SCEPTOR aircraft proceeded into two phases. First, for a given number of propellers, many different high-lift propeller designs were evaluated and a preferred design selected. Second, the preferred designs for each number of propellers were compared to one another to determine an overall preferred configuration and propeller design. Following this process has enabled insight into the design space and the sorts of tradeoffs that may be expected in high-lift propeller system design.

There are many tradeoffs that designers must consider when selecting the general configuration layout of an aircraft with high-lift propellers. Because there are many competing metrics, it is unlikely that there will be an abundantly clear "optimum" number of high-lift propellers to select for any given configuration. Both nominal performance and performance in off-nominal situations such as the loss of one high-lift motor should be considered in selecting the number of propellers for a configuration.

The propeller design method and the number of blades selected for the prop design may have large impacts on the performance of the high-lift system and, consequently, the appropriate number of high-lift propellers that should be selected. This indicates that simple momentum theory or other methods that are agnostic to the number of blades are likely to result in an unrealistic picture of the design space and the selection of a non-optimal number of propellers for the configuration.

A potential constraint on the design of the high-lift propellers system is the total thrust. If large amounts of lift augmentation are required from the propellers, then high thrust values from the high-lift propellers are likely. If these propellers produce excess thrust, then the aircraft will be unable to sustain flight at the desired speed. This implies that drag producing devices may need to be added to the aircraft or the primary propellers operated as windmills to produce additional drag.

\section{Acknowledgments}

This work was funded under the Convergent Aeronautics Solutions and Transformational Tools and Technologies Projects of the Transformative Aeronautics Concepts Program. We would like to thank the entire SCEPTOR team including those at NASA Langley, NASA Armstrong, NASA Glenn, Empirical Systems Aerospace (ESAero), and Joby Aviation for their input and support as well as their own work 
on this project.

\section{References}

${ }^{1}$ Moore, M. D. and Fredericks, B., "Misconceptions of Electric Aircraft and their Emerging Aviation Markets," 52nd Aerospace Sciences Meeting, 2014, AIAA 2014-0535.

${ }^{2}$ Miranda, L. R. and Brennan, J. E., "Aerodynamic Effects of Wingtip-Mounted Propellers and Turbines," 4th Applied Aerodynamics Conference, San Diego, CA, June 9-11 1986, pp. 221-228, AIAA-86-1802.

${ }^{3}$ Snyder, M. and Zumwalt, G., "Effects of Wingtip-Mounted Propellers on Wing Lift and Induced Drag," Journal of Aircraft, Vol. 6, No. 5, 1969, pp. 392-397.

${ }^{4}$ Veldhuis, L. L. M., Propeller Wing Aerodynamic Interference, Ph.D. thesis, Delft University of Technology, June 2005.

${ }^{5}$ Koning, C., Aerodynamic Theory, Vol. IV, Div. M, chap. Influence of the Propeller on Other Parts of the Airplane Structure, Julius Springer, Berlin, 1935, pp. 361-430.

${ }^{6}$ Franke, A. and Weinig, F., "The Effect of Slipstream on an Airplane Wing," NACA-TM-920, National Advisory Committee for Aeronautics, 1939.

${ }^{7}$ Squire, H. B. and Chester, W., "Calculation of the Effect of Slipstream on Lift and Induced Drag," Reports and Memoranda 2368, British Aeronautical Research Council, 1950.

${ }^{8}$ Mcveigh, M. A., Gray, L., and Kisielowski, E., "Prediction of Span Loading of Straight-Wing/Propeller Combinations Up to Stall," Nasa-cr-2602, National Aeronautics and Space Administration, October 1975.

${ }^{9}$ Rethorst, S., "Aerodynamics of Nonuniform Flows as Related to an Airfoil Extending Through a Circular Jet," Journal of the Aeronautical Sciences, Vol. 25, No. 1, 1958, pp. 11-28.

${ }^{10} \mathrm{Wu}, \mathrm{T}$. Y. and Talmadge, R. B., "A Lifting Surface Theory for Wings Extending Through Multiple Jets," Tech. Rep. 8, Vehicle Research Corp., Pasadena, California, 1961.

${ }^{11}$ Ribner, H. and Ellis, N., "Theory and Computer Study of a Wing in a Slipstream," 4th Aerospace Sciences Meeting, Los Angeles, CA, June 27-29 1966, AIAA 66-466.

${ }^{12}$ Patterson, M. D. and German, B. J., "Simplified Aerodynamics Models to Predict the Effects of Upstream Propellers on Wing Lift," 53rd AIAA Aerospace Sciences Meeting, Kissimmee, Florida, 5-9 January 2015, AIAA 2015-1673.

${ }^{13}$ Ting, L. and Liu, C., "Thin Airfoil in Nonuniform Parallel Streams," Journal of Aircraft, Vol. 6, No. 2, 1969, pp. 173-175.

${ }^{14}$ Chow, F., Krause, E., Liu, C. H., and Mao, J., "Numerical Investigations of an Airfoil in a Nonuniform Stream," Journal of Aircraft, Vol. 7, No. 6, 1970, pp. 531-537.

${ }^{15}$ Ting, L., Liu, C. H., and Kleinstein, G., "Interference of Wing and Multipropellers," AIAA Journal, Vol. 10, No. 7, 1972, pp. 906-914.

${ }^{16}$ Nichols, R., Tramel, R., and Buning, P., "Solver and Turbulence Model Upgrades to OVERFLOW 2 for Unsteady and High-Speed Applications," 24th AIAA Applied Aerodynamics Conference, Fluid Dynamics and Co-located Conferences, San Francisco, CA, June 5-8 2006, AIAA 2006-2824.

${ }^{17}$ Nichols, R. and Buning, P., User's Manual for OVERFLOW 2.2, NASA Langley Research Center, Hampton, VA, August 2010.

${ }^{18}$ Patterson, M. D., Conceptual Design of High-Lift Propeller Systems for Small Electric Aircraft, Ph.D. thesis, Georgia Institute of Technology, August 2016, [upcoming publication].

${ }^{19}$ Borer, N. K., Moore, M. D., and Turnbull, A., "Tradespace Exploration of Distributed Propulsors for Advanced OnDemand Mobility Concepts," 14th AIAA Aviation Technology, Integration, and Operations Conference, Atlanta, GA, 16-20 June 2014, AIAA 2014-2850.

${ }^{20}$ Patterson, M. D., Borer, N. K., and German, B. J., "A Simple Method for High-Lift Propeller Conceptual Design," 54 th AIAA Aerospace Sciences Meeting, AIAA SciTech, San Diego, California, 4-8 January 2016, AIAA 2016-0770.

${ }^{21}$ Borer, N. K., Patterson, M. D., Stoll, A. M., Derlaga, J. M., and Moore, M. D., "Design and Performance of the NASA SCEPTOR Distributed Electric Propulsion Flight Demonstrator," AIAA Aviation 2016, Washington, D.C., June 13-17 2016, [upcoming publication].

${ }^{22}$ Hepperle, M., "MH 114," http://www.mh-aerotools.de/airfoils/ mh114koo.htm, 16 Feb 2008, accessed 5 Nov 2015.

${ }^{23}$ Joby Motors, "Products," http://www.jobymotors.com/public/views/ pages/products.php, 2011, accessed 16 Oct., 2015.

${ }^{24}$ Raymer, D. P., Aircraft Design: A Conceptual Approach, American Institute of Aeronautics and Astronautics, 4th ed., 2006.

${ }^{25}$ Hahn, A. S., "Vehicle Sketch Pad: A Parametric Geometry Modeler for Conceptual Aircraft Design," 48th AIAA Aerospace Sciences Meeting Including the New Horizons Forum and Aerospace Exposition, Orlando, Florida, January 4-7 2010, AIAA-2010-657.

${ }^{26}$ OpenVSP, http://www.openvsp.org/, accessed 28 Aug 2012.

${ }^{27}$ Drela, M. and Youngren, H., "XROTOR Download Page," http://web.mit.edu/drela/Public/web/xrotor/, accessed 26 May 2014.

${ }^{28}$ Larrabee, E. E., "Practical Design of Minimum Induced Loss Propellers," SAE Technical Paper 790585, Society of Automotive Engineers, 1979.

${ }^{29}$ Larrabee, E. E. and French, S. E., "Minimum induced loss windmills and propellers," Journal of Wind Engineering and Industrial Aerodynamics, Vol. 15, December 1983, pp. 317-327. 